\title{
LOG TRANSFORM BASED OPTIMAL IMAGE ENHANCEMENT USING FIREFLY ALGORITHM FOR AUTONOMOUS MINI UNMANNED AERIAL VEHICLE: AN APPLICATION OF AERIAL PHOTOGRAPHY
}

\author{
SOURAV SAMANTA \\ Department of Computer Science \& Engineering, University Institute of Technology, Burdwan, Westbengal, \\ INDIA \\ sourav.uit@gmail.com \\ AMARTYA MUKHERJEE \\ Institute of Engineering and Management, Kolkata, Westbengal, INDIA \\ mamartyacse1@gmail.com \\ AMIRA S. ASHOUR \\ Department of Electronics \& Electrical Communications Engineering, Faculty of Engg., Tanta Univ., EGYPT. \\ College of Computers \& IT, Taif University, KSA. \\ amirasashour@yahoo.com \\ NILANJAN DEY \\ Dept. of CSE, Bengal College of Engineering and Technology, Durgapur, INDIA \\ neelanjan.dey@gmail.com \\ JOÃO MANUEL R. S. TAVARES \\ Instituto de Ciência e Inovação em Engenharia Mecânica e Engenharia Industrial, Departamento de \\ Engenharia Mecânica, Faculdade de Engenharia, Universidade do Porto, Rua Dr. Roberto Frias, s/n, 4200- \\ 465 Porto, PORTUGAL \\ tavares@fe.up.pt \\ WAHIBA BEN ABDESSALEMKARAA \\ College of Computers \& IT, Taif University, KSA. RIADI-GDL laboratory, TUNISIA \\ wahiba.bak@gmail.com \\ REDHA TAIAR \\ Université de Reims Champagne Ardennes, FRANCE \\ redha.taiar@univ-reims.fr \\ AHMAD TAHER AZAR \\ Faculty of Computer and Information, Benha University, EGYPT \\ ahmad_t_azar@ieee.org \\ ABOUL ELLA HASSANIEN \\ Cairo University, Scientific Research Group, EGYPT \\ aboitcairo@gmail.com
}

The Unmanned Aerial Vehicles (UAV) are widely used for capturing images in border area surveillance, disaster intensity monitoring, etc. An Aerial photograph offers a permanent recording solution as well. But rapid weather change, low quality image capturing equipments results in low/poor contrast images during image acquisition by Autonomous UAV. In this current study a well known meta-heuristic technique, namely, Firefly Algorithm (FA) is reported to enhance aerial images taken by a Mini Unmanned Aerial Vehicle (MUAV) via optimizing the value of certain parameters. These parameters have a wide range as 
used in the Log Transformation for image enhancement. The entropy and edge information of the images used as an objective criterion for evaluating the image enhancement of the proposed system. Inconsistent with the objective criterion, the FA is used to optimize the parameters employed in the objective function that accomplishes the superlative enhanced image. A low light imaging has been performed at evening time to prove the effectiveness of the proposed algorithm. The results illustrate that the proposed method has better convergence and fitness values compared to Particle Swarm Optimization. Therefore, FA is superior to PSO, as it converges after a less number of iterations.

Keywords: Mini Unmanned Aerial Vehicle (MUAV); Meta-heuristic approaches; Particle Swarm Optimization (PSO); Firefly algorithm; Log Transformation; Aerial Photography.

\section{Introduction}

An Unmanned Aerial Vehicle (UAV) is defined as a space traversing vehicle that flies without a human team on board and can be remotely controlled or fly separately [1]. In the recent era, the application of the UAV for surveillance, aerial photography, crowd monitoring, industrial infrastructure monitoring and locate human survivors in a disaster hit region have a paramount significance. Most of the cases are the "Birds Eye View" images that are captured with the equipments like smaller CMOS sensor based camera, surveillance camera equipments. There are factors that affect the image contrast named as:

1- Bad weather conditions like heavy rainfall, dense fog and storm. Most of the images captured by such units become low contrast.

2- The change in flight altitude due to rapid waypoint navigation, GPS glitch and lack of proper night vision.

The image captured by those camera units becomes blurred and hazy. Therefore, both the objects and activity tracking have almost been impossible from these captured images.

In addition, the contrast of aerial images may vary with the changes in sunlight angle. As an aerial vehicle having limited payload capacity, therefore, it is difficult to carry a high end image capturing equipments by the aerial vehicle especially for Mini UAV.

Consequently, the lens quality and the focal length of the camera become compromised, which affects the image contrast.

As observed several of the high end image capturing unit fails to capture high quality images under such situation. As a result of these circumstances a proper optimized image enhancement algorithms are obviously necessary. Image enhancement is used for improving the perception of information in images as well as achieving superior input for another image processing automated techniques.

The main objective of aerial image enhancement is to modify attributes of any aerial image as per the given task. Moreover, image enhancement depends on precise factors, such as the viewer's experience and the human visual system. For digital image enhancement without distortion, variety of techniques can be used. There are two categories for the enhancement methods which are:

1- Spatial Domain Method where the pixel values are controlled to attain the preferred image enhancement.

2- Frequency Domain Method as, the image is first transferred to frequency domain. These enhancement operations are performed in order to modify the image brightness, contrast or the distribution of the gray levels. As a result of the transformation function 
applied to the input values, the pixel value (intensities) of the output image gets updated accordingly. Image enhancement is applied in various fields where images are analyzed and enhanced using various algorithms.

A variety of algorithms are anticipated to solve the complicated multi constrained optimization problem for image enhancement. Where, optimization is a mathematical discipline concerns finding of minima and maxima of functions, subject to constraints.

Optimization algorithms which solve such problems are differential evolution [2], biogeography-based optimization [3], genetic algorithm [4], ant colony algorithm [5] and its variant [6], Cuckoo search [7], Honey-Bees Mating Optimization (HBMO) Algorithm [8], Particle Swarm Optimization (PSO) [9], Firefly algorithm [10], etc.

These heuristic and meta-heuristic methods are used to enhance or improve images in some sense. Additionally, robust image acquisition for aerial platforms is introduced to mimic a real life mission critical image grabbing, processing or enhancement. Accordingly, the objective functions are used for image enhancement method. In this work, a very popular meta-heuristic algorithm, Firefly Algorithm is used to optimize the parameters of the Log transformation to enhance the images taken by a MUAV.

\section{RELATED WORK}

Various studies have been done so far on image enhancement and UAV based image processing domain. In [11] a first proposed image enhancement and thresholding by the optimization of fuzzy compactness was introduced. Where, an algorithm was proposed based on minimization of fuzzy compactness to check whether it is possible to obtain both fuzzy and non-fuzzy versions of any ill-defined image. An automatic image enhancement technique using genetic algorithms (GA) was proposed by [12]. The GA was used to enhance the contrast and detail in the image according to an objective fitness criterion. Proposed image magnification technique using partitioned iterative function system was introduced [13]. In the suggested work, genetic algorithm was used to enhance the contrast and gray-level-modification of an image. A video Geo-location and image enhancement technique for small unmanned air vehicles was discussed [14]. As a low cost GPS and attitude sensor to estimate the object position on the ground and a simple super-resolution technique was used for image enhancement. Through [15], an image enhancement technique using Particle Swarm Optimization for image processing and optimization was proposed. In [16], a Map-Enhanced UAV Image Sequence Registration and Synchronization of Multiple Image Sequences were offered. A grayscale image enhancement technique using Particle Swarm Optimization was suggested [17]. Where, image enhancement was considered as an optimization problem, and image enhancement is done via Particle Swarm Optimization. A novel approach for image edge enhancement using Ant Colony Optimization algorithm using Hybridized Smoothening Filters was introduced [18]. The Ant colony optimization was used for image edge enhancement. In [19], an image enhancement technique using Fuzzy system inference was suggested. Since, this technique was proposed using Fuzzy system, which was able to enhance the contrast of an image. This algorithm was used to convert image properties into fuzzy data and fuzzy 
data into defuzzification. A novel image enhancement method using Particle Swarm Optimization was proposed [20]. An image filter based on PSO was adopted to eliminate noise and the image enhancement was presented using the PSO and DWT to improve the image quality. In [21], a Non-linear Grayscale Image Enhancement technique based on Firefly Algorithm as an adaptive local enhancement algorithm was introduced. A new Image Enhancement technique using Ant Colony Optimization as scaling factors for optimization was established in [22]. The scaling factors were pixel assessment, cluster mean update and threshold calculation. In [23], a novel Quantum-behaved PSO with an Adaptive Strategy (AQPSO) was proposed for enhancement of the grayscale as well as the color images. A PSO technique for image enhancement was proposed. This PSO was based on hue preserving color image enhancement technique [24].

The ultimate goal of our image enhancement methodology is to present a complete prototype test platform to grab a low contrast; aerial image at evening time and after grabbing the image, Firefly based image enhancement algorithm is applied. In advance, similar type of work is reported in [21] for image enhancement. Also, a Log Transform based image enhancement technique was proposed in [25] for processing Multiple Biometric Grand Challenge (MBGC) iris data. This results in significant improvement in the performance of iris recognition algorithms in terms of equal error rates.

The motivation of our work is to find the optimal parameter's value used in Log Transform for enhancement of each image. The parameter optimization is done through FA based enhancement technique in real time applications to study the behavior of the aerial images taken by the UAV. Also, the FA results are compared with the PSO for various gray images.

\section{IMAGE ACQUISITION USING UAV}

The UAV system used for data acquisition is a mostly low cost system and flexible. Therefore, it can be considered as a suitable alternative solution compared to other mobile mapping systems. Mainly, a stable Multi-rotor Mini UAV is chosen in this work; to achieve the major objective of enhancing the mission critical image information.

A multirotorcraft offers great advantages in reconnaissance and aerial surveillance. Design of a MUAV is a challenge theoretically and technically as well. The multirotor system used in this work follows the Newtonian model of a six rotor multicopter. The hexcopter is assumed to be a rigid body system, having 6 degrees of freedom. The motion of the system can be obtained by increasing the speed of each rotor in various fashions. Generally a vertical linear motion can be obtained by increasing and decreasing the speed of each motor simultaneously. The forward and backward motion can be obtained by increasing the speed of the rear and front motors respectively (known as a pitch). Whereas right and left side movement can be obtained by differentially controlling the left and right side motors respectively (known as roll). A yaw movement can also be achieved by differentially controlling all six motors by considering the total thrust is constant.

The Multi-rotor system has exclusive features as it can:

- Holds any altitude and location constantly. 
- Monitors from that position towards its $360^{\circ}$ visibility range by controlling its heading within that constant position.

During this mission; a flight route, though which the UAV have to navigate and at a particular altitude and location is created. It holds its position and performs the image acquisition task. The images have been taken with:

1- 1 st image with a heading of $0^{\circ}$;

2- 2nd image has been grabbed with a heading value of $45^{\circ}$;

3- 3rd image has been captured with a heading of $90^{\circ}$;

4- 4th image has been taken with a heading of $180^{\circ}$.

To prove the effectiveness of the proposed algorithm, a low light imaging has been performed at evening time.

\subsection{Image Acquisition Steps}

To perform successful acquisition task, the step of commands has been fed to the MUAV (mini-UAV).

1- At the initial startup of flight, the MUAV performs a stabilize mode take off. In this mode the control is performed by the ground station situated at the home location.

2- After 45 seconds of stabilizing mode flight, the UAV moves to autonomous mode and performs auto navigation, loiter and return to home operation in a sequence.

3- Loiter (Position Hold) mode flight [26] is the more suitable to take an aerial image because it performs a fixed location hold operation based on GPS and altitude lock. Figure 1 shows the instruction execution sequence of the MUAV for performing aerial photography.

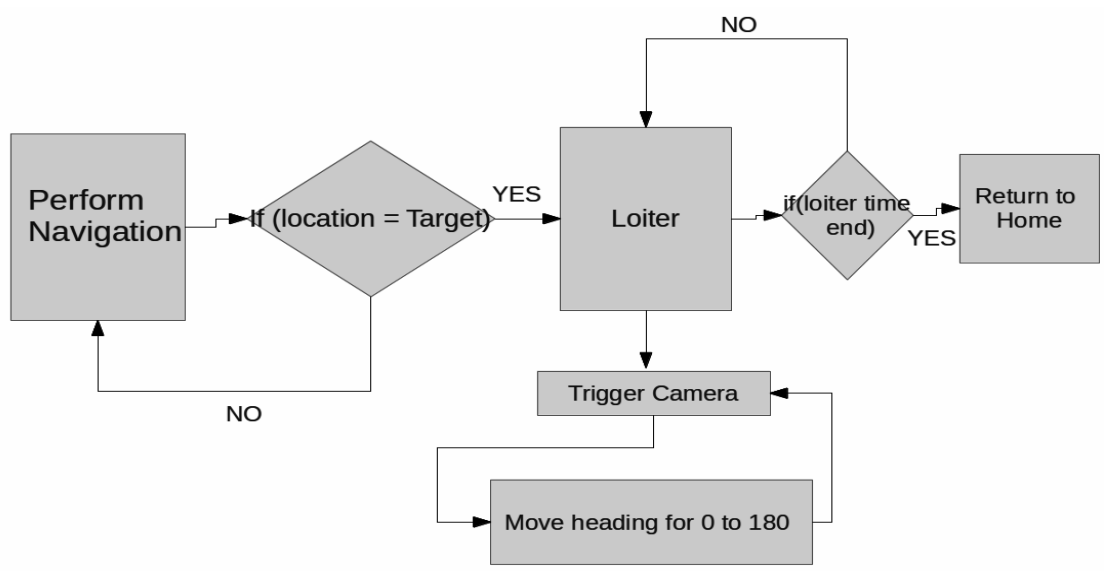

Fig. 1. Block Diagram of image acquisition

4-The auto navigation is performed until the desired target waypoint achieved.

5-As the UAV reaches the target waypoint it initiates loiter mode where it becomes stationary in a particular location. 
6-Now, the image acquisition task is started by triggering the camera at $0^{\circ}$ heading to $180^{\circ}$ heading.

7-As the loiter interval expires, the UAV has to return to the base.

Here the waypoint navigation has been done on the basis of the optimized DCM algorithm which computes the attitude of the flight with respect to the yaw, pitch and roll. The equilateral projection methodology has been used to compute the next waypoint distance by using the bearing value obtained from magnetic compass and the GPS latitude and longitude. The guidance and navigation logic use here is mainly depends upon a high level decision making. The basic duty of the system is to generate the trajectory and planning of the path. The programming logic implemented has basically supported multi threading that offers parallel action of different instruction execution and sensor data fusion.

\subsection{Ground Control Station Responsibility}

The ground control station is one of the active key elements that perform the critical task of command, control and data analysis in real time. A standard GCS system generally consists of the ground station computer that runs an open source, OpenGL based software, a navigation control unit and a real time telemetry unit. The telemetry system uses $433 \mathrm{MHz}$ radio band that connects the UAV to ground station with a baud rate of 56700 bps. Stick command has been transmitted to the UAV unit using $2.4 \mathrm{GHz}$ PWM signal. Typically 5 channels have assigned the UAV to control the flight from the ground station are roll, pitch, yaw and flight mode. The primary task of the radio command module is to handle the fail safe operation while in flight. The telemetry units have full duplex communication capability through which the command can be sent from the ground station and the viceversa.

\subsection{Image Acquisition Challenges}

The launched aerial vehicular platform has several challenges that are to be taken into account to grab high precision image information of a target point. There are some of the inherent challenges regarding to this context are:

a) The limited flight time that cause limited amount of image information.

b) The environmental changes affects directly to the attitude of the UAV causes serious performance overhead in some cases.

Although, the UAV systems are well tested before deployment there exist a chance of link failure caused by:

- The ground station;

- Improper position, hold due to GPS glitch;

- Improper compass heading due to external electromagnetic interference.

Such reasons degrade or even generate a malfunction on the UAV systems.

Other indirect challenges are mentioned as follows:

i. Image distortion might occur due to the unwanted vibration and oscillation of the camera gimbal unit.

ii. Roll, Pitch, Yaw change of the UAV might affect the location accuracy of the image. 
The MUAV platform is assumed to be aerial maneuverability and reachability chosen for its easy deploys ability.

The main goal of this work is to use the Log Transform with Firefly optimization algorithm for enhancing the Autonomous Mini Unmanned Aerial Vehicle's Image.

Therefore, in the next section a transform function is discussed.

\section{FUNCTION OVERVIEW}

\subsection{Transformation Function}

Wherever, local enhancement model applies the transformation functions that are based on the gray-level allocation in the neighborhood of each pixel in the input image. The intensity value for each pixel of the input image will be taken by the transformation function to produce a new intensity value for the related pixel to construct the enhanced image. Image enhancement can be done through gray level transformations which has three basic types, Linear, Logarithmic or Power-law.

In the conventional enhancement technique, enhancement occurs at each pixel at location $M \times N$ of the original image. $M$ denotes the number of columns and $N$ denotes the number of rows. The enhancement can be illustrated in [21],

$$
g(i, j)=T[f(i, j)]
$$

Where $f(i, j)$ is the input image gray value pixels $(i, j)$ and $g(i, j)$ is the gray value of the enhanced image pixels $(i, j)$, while $T$ is the transformation function.

To extract the local information, a window of size $n \times n$ is used. The transformation is defined as follows:

$$
g(i, j)=K(i, j)[f(i, j)-c \times m(i, j)]+m(i, j)^{a}
$$

Here, $a$ and $c$ are parameters.

The local mean of the $(i, j)^{\text {th }}$ pixel of the input image over a $n \times n$ window is,

$$
m(i, j)=\frac{1}{n \times n} \sum_{x=0}^{n-1} \sum_{y=0}^{n-1} f(x, y)
$$

$K(i, j)$ is the enhancement function, which considers both the local and global information, and has the form:

$$
K(i, j)=\frac{k \cdot D}{\sigma(i, j)+b}
$$

Where $k$ and $b$ are two parameters.

The global mean is defined as:

$$
D=\frac{1}{M \times N} \sum_{i=0}^{M-1} \sum_{j=0}^{N-1} f(i, j)
$$


In addition to the local standard deviation of the input image pixel $(i, j)^{t h}$ of over a $n \times n$ window, is given as:

$$
\sigma(i, j)=\sqrt{\frac{1}{n \times n} \sum_{x=0}^{n-1} \sum_{y=0}^{n-1}(f(x, y)-m(i, j))^{2}}
$$

Substitute from equation 4 in equation 2 to get the new transformation function as:

$$
g(i, j)=\frac{k \cdot D}{\sigma(i, j)+b}[f(i, j)-c \times m(i, j)]+m(i, j)^{a}
$$

Through equation (7), the contrast of the image is stretched, where $m(i, j)$ is the center of the stretch. The four parameters mentioned before are to produce large variations in the input image.

In order to enhance the image, as the overall contrast and the sharpness has to be increased, Lee's algorithm with the log transform is used.

\subsection{Logarithmic Image Processing (Log Transform)}

In [25] a modified method for the implementation of Lee's algorithm was shown its facility to produce good quality enhanced. Foremost, for an 8-bit image $(\mathrm{L}=256)$, the image intensity function $F$ is transformed to the gray tone function as:

$$
f: F(i, j)=L-f(i, j)
$$

Afterward, a normalized complement transform is done where; the gray tone function is transformed to the normalized negative gray tone function through:

$$
\bar{f}=1-\frac{f}{L}
$$

Subsequently, the logarithm of equation (9) is applied using Lee's Algorithm [25] as:

$$
\log \left(\bar{f}^{\prime}(i, j)\right)=\lambda \log (\bar{a}(i, j))+\mu[\log (\bar{f}(i, j))-\log (\bar{a}(i, j))]
$$

Where, the mean value of $\bar{f}$ in a window of $\operatorname{size}(\eta \times \eta)$ centered on $(i, j)$ is $\bar{a}(i, j)$. By converting $f^{\prime}$ back to the original scale, the enhanced output image $F^{\prime}$ is obtained.

From equation 10, the three control parameters for the algorithm are:

$1-\lambda$ is used to modify the dynamic range of an image. The smaller the value of $\lambda$, the brighter the image will appear.

$2-\mu$ is used to control the sharpness of an image. Where, the greater value of $\mu$ leads to a sharper image. Its range is $\mu \in[0,255]$.

$3-\eta$ is used to control the window size.

An initial range of $[0,1.5]$ is used for both $\lambda$ and $\mu$, while $\eta$ is chosen to be 3 for the proposed UMV aerial image application through experiment. Then, the selection of the exact value of $\lambda$ and $\mu$ to enhance the dark aerial image within the given range is done by Firefly algorithm to get the optimal parameter's value, which leads to enhanced image. Enhancing the quality of an image is described in the next section. 


\section{EVALUATION CRITERIA}

An objective function is required to evaluate and measure the quality of the enhanced image. Three performance measures will be used to produce the objective function:

- Entropy value;

- Edge intensities sum; and

- Number of edge pixels.

A good enhanced image has more number of edges and higher intensity of edges as compared to the original image. However, the entropy value of the image is introduced, as only two measures are not enough to test the image enhancement. The entropy is chosen as its value contains information about the image. The objective function [21] considered here is:

$$
F\left(I_{e}\right)=\log \left(\log \left(E\left(I_{s}\right)\right)\right) \times \frac{n_{\text {edges }}\left(I_{s}\right)}{M \times N} \times H\left(I_{e}\right)
$$

Where $I_{e}$ is the enhanced image created using the transformation function in equation (2); while, $I_{s}$ is the edge image using Sobel edge detection, which is one of the efficient automatic threshold detector algorithms. $E\left(I_{s}\right)$ is sum of pixel intensities of the Sobel edge image $I_{s}$ and $n_{\text {edges }}$ is the number of pixels, whose intensity value is above a threshold in $I_{s}$.

Using the enhanced image $I_{e}$ to produce an edge image is as:

$$
I_{s}(i, j)=\sqrt{\delta m I_{e}(i, j)^{2}+\delta n I_{e}(i, j)^{2}}
$$

Where,

$$
\begin{aligned}
\delta m I_{e}(i, j)= & g I_{e}(i+1, j-1)+2 g I_{e}(i+1, j)+g I_{e}(i+1, j+1)-g I_{e}(i-1, j-1)-2 g I_{e}(i-1, j)-\ldots \\
& g I_{e}(i-1, j+1) \\
\delta n I_{e}(i, j)= & g I_{e}(i-1, j+1)+2 g I_{e}(i, j+1)+g I_{e}(i+1, j+1)-g I_{e}(i-1, j-1)-2 g I_{e}(i, j-1)-\ldots \\
& g I_{e}(i+1, j-1)
\end{aligned}
$$

Since, the histogram transformation is thought to be one of the fundamental processes for contrast enhancement of gray level images; therefore, the entropy value is estimated from the enhanced image $I_{e}$, by applying on the histogram as:

$$
H\left(I_{e}\right)=\sum_{i=0}^{255} h_{i} \log _{2}\left(h_{i}\right)
$$

As $h_{i}$, is the probability of occurrence of the $i^{\text {th }}$ intensity value of $I_{e}$ image.

\section{FIREFLY ALGORITHM BASED IMAGE ENHANCEMENT}

A nature stimulated meta-heuristic algorithm was developed in [27, 28], namely Firefly Algorithm (FA). In order to improve the global search and local optimization ability, the Firefly optimization algorithm will be used. FA is used to search the optimal parameters 
for the best enhancement. It is a non-gradient based, considered as a simple objective function that can produce a valuable result, while dealing with the highly nonlinear dynamic optimization problems. Because of the gradient evaluations, these optimization problems can be solved by avoiding bad numerical behavior. The FA is also able to find the optimal solution to a problem by iteratively enhancing a candidate solution, with the provided measure of solution quality. The FA is like all other popular meta-heuristic algorithm for optimization. Firefly algorithm is based on the idealized behavior of the flashing feature of fireflies. The following three rules are stated for the basic formulation of FA as presented in [29]:

- Fireflies are unisexual. Therefore, they move towards more appealing and brighter fireflies irrespective of their sex.

- Attractiveness is proportional to their brightness, which is inversely proportional to the distance between two flies.

- The less bright flashing firefly moves towards the brighter one. A firefly moves randomly, if there are no brighter fireflies than the particular one.

The landscape of the objective function determines the brightness of a firefly. In most of the problem domains, the value of the objective function is proportional to brightness. Since, the firefly's attractiveness is related to the light intensity seen by adjacent fireflies, hence the variation of attractiveness $\beta$ with the distance $r$ can be defined by:

$$
\beta=\beta_{0} e^{-\gamma r^{2}}
$$

The movement of a firefly $i$ is attracted to another more attractive (brighter) firefly $j$ is established by:

$$
x_{i}^{t+1}=x_{i}^{t}+\beta_{0} e^{-\gamma r_{i j}^{2}}\left(x_{j}^{t}-x_{i}^{t}\right)+\alpha_{t} \varepsilon_{i}^{t}
$$

Where:

- $2^{\text {nd }}$ term is due to the attraction. If $\beta_{0}=0$; it becomes a simple random walk.

- $3^{\text {rd }}$ term is randomization with $\alpha_{t}$ being the randomization parameter. This parameter can vary with the iteration counter $t$ in order to be adjusted during iterations.

$\varepsilon_{i}^{t}$ is a vector of random numbers drawn at time $t$.

In fact, the PSO variants are considered as a special case of Firefly algorithm, when $\gamma=0$.

The FA several major advantages over other algorithms, for example:

- It has automatically subdivision property, as FA is based on attraction and attractiveness decreases with distance. Therefore, the entire population can be subdivided into subgroups. Every group can swarm around each mode or local optimum. The finest global solution can be obtained through all these modes.It can deal with multimodality. Where, if the population size is adequately higher than the mode numbers, the subdivision will allocate the fireflies in a manner so as to be able to find all optima simultaneously. 
- The parameters in FA can be adjusted to control the randomness as the iterations proceed, so that convergence can be speed up by tuning these parameters.

These advantages make the FA flexible to deal with continuing problems, classifications and combinatorial optimization as well.

Thus, the Firefly Optimization Algorithm is a framework to optimize the grayscale values of the image. FA is used to find the optimal values of the three parameters in the log transformation, where they have a wide range.

\section{PROPOSED METHOD}

Prior studies show that, in most cases firefly algorithm gives better convergence towards global optimization than any other global search algorithm. Parameter optimization in log transformation based image enhancement technique using firefly algorithm is introduced in this work. According to equation (10), $\lambda, \mu$ are the two parameters that have to be optimized. While, the third parameter, window size control; $\eta$ will set to 3 .

In the proposed method, the following assumptions and steps will be tracked:

1-Each firefly is represented by $\lambda$ and $\mu$, clearly each one belongs from four dimensional space ( $d=4$ where $d$ define the dimensions of firefly).

2-The range of parameters $\lambda$ and $\mu$ has taken within the range of [0 to 1.5].

3 -The numbers of fireflies is 10 .

4-Each firefly is used, to enhance the original image using equation (10).

5 -Light intensity of each firefly is determined.

6-After every iteration, all fireflies move towards the firefly with high light intensity and light absorption is also updated.

7-When algorithm reaches its maximum iteration, it stops and gives the global optimum solution containing values of $\lambda_{g}$ and $\mu_{g}$. These are the optimized values by the firefly algorithm.

The Firefly Algorithm for the optimization of the Log transform parameters is:

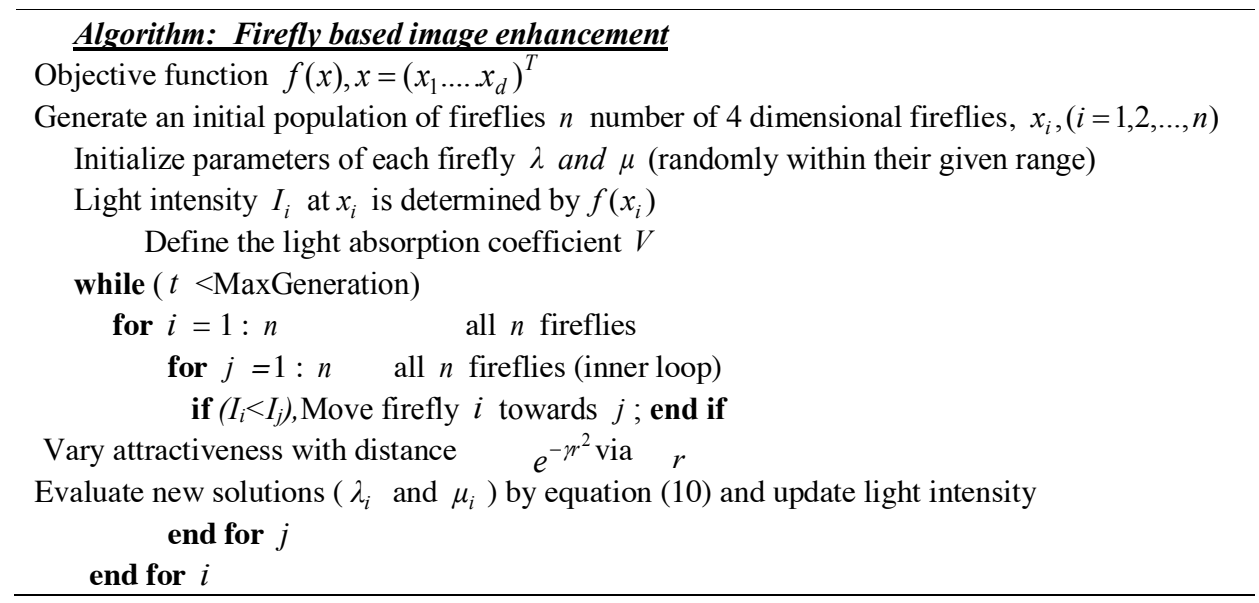


Rank the fireflies and find current global best $\mathrm{g}\left(\lambda_{g}\right.$ and $\left.\mu_{g}\right)$.

end while

Post-process results and visualization of enhanced image

\section{EXPERIMENTAL SETUP}

The Hex-rotor system is supposed to be the best MUAV platform for critical image acquisition. The reason behind choosing hex-rotor architecture is in order to:

1- Achieve more aerial stability while taking the air borne image.

2- Offer more payload capacity amongst any other UAV systems.

For this work, a scratch built UAV where:

* The UAV is made of on board Gyro, Barometer, an external GPS and Magnetic Compass to perform the most efficient position hold and loiter operation.

* The system used an ATMega 2560 Microcontroller based autopilot unit.

* The frame is made of fiberglass with $550 \mathrm{~mm}$ width and $60 \mathrm{~mm}$ height (including Landing Gear).

*The image has been taken with a 2 mega pixel $1.6 \mathrm{~mm}$ hard mount the camera at an altitude of 18 meters.

* Loiter mode flight operation has been chosen to stabilize the image acquisition event.

* The Ground Station has two parts; one consists of three subsystem:

i. A ground station computer that runs APM mission planner v1.2.95 to set up the flight plan and store $433 \mathrm{MHz}$ telemetry data. The data interface communication has been done with a baud rate of 56700 bps.

ii. A $2.4 \mathrm{GHz} 9$ channel radio controller performs manual roll, pitch, yaw, throttle and flight mode control.

iii. A 5.8 GHz image Transmitter transmits the captured image form UAV to Base Station.

Hence the image has been stored at the base station computer. In the prototype mission, we are trying to mimic a maximum realistic scenario as if the UAV gets crashed during mission, still we can successfully retrieve the images at the base station. Figure 2 shows the Ground Station software, MUAV unit, Ground Station Units and on flight UAV; respectively. The ground station software here displays the waypoint information corresponding to image acquisition event starting from the home to $1,3,5,7$; respectively. 


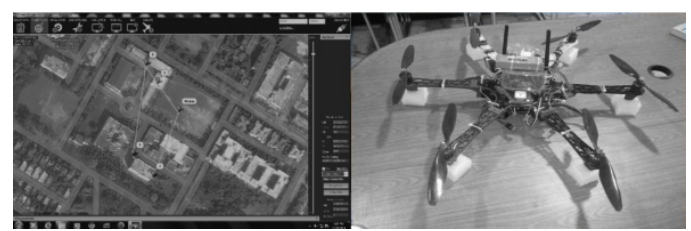

(a)

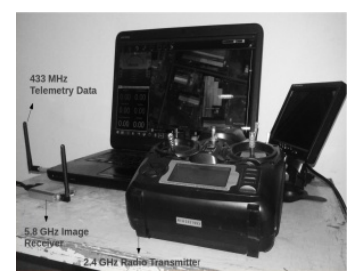

(c) (b)

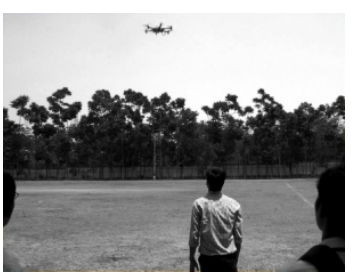

(d)

Fig.2. (a) Ground Station software, (b) MUAV unit, (c) Ground Station Units, (d) On flight UAV

The auto navigation speed of the MUAV has set to $200 \mathrm{~cm} / \mathrm{sec}$ to avoid any instability due to wind resistance. The entire flight has been done within the three way point (Home, Waypoint 1 , Waypoint 2). Compass bearing is set to $0^{\circ}$ for straight flight orientation. Wrong orientation of the compass may cause an abnormal circular flight. Loiter time signifies that waypoint where the photographs has been captured the MUAV must wait for 120 seconds. Return to home altitude is set for final altitude value at the time of returning to the home location. As more stable UAV takes more precise images, PID Gain value corresponding loiter mode has a great significance too. Proportional value $\mathrm{P}$ set to 1.000 signifies that the position holds an error is to be compensated with $100 \%$ precision which makes smoother position hold compensation. Integral value I set to 0.5000 signifies $50 \%$ of heading hold capability where as value D sets to 0.010 signifies how fast UAV reacts when position error occurs. The higher value of this parameter makes a huge oscillation. Therefore the value must set as minimum as possible. Here the gain values have been set such that the MUAV gives its maximum precise Loiter performance while capturing the images. Flight specific parameter settings and the MUAV hardware specification are tabulated in Tables 1 and 2; respectively. 
Table 1. Flight specific parameters

\begin{tabular}{|c|c|c|}
\hline Flight Parameter & \multicolumn{2}{|l|}{ Value } \\
\hline Navigation Speed & \multicolumn{2}{|l|}{$200 \mathrm{~cm} / \mathrm{Sec}$} \\
\hline Number of WP & \multicolumn{2}{|l|}{3} \\
\hline NAV Altitude & \multicolumn{2}{|l|}{18 meters } \\
\hline Compass Orientation & \multicolumn{2}{|l|}{$0^{\circ}$} \\
\hline AHRS GPS Use & \multicolumn{2}{|l|}{1 (yes) } \\
\hline Waypoint Loiter Time & \multicolumn{2}{|l|}{$120 \mathrm{sec}$} \\
\hline Return to Home Altitude & \multicolumn{2}{|l|}{3 meters } \\
\hline \multirow[t]{3}{*}{ Loiter Gain value } & $\mathrm{P}$ & 1.000 \\
\hline & I & 0.500 \\
\hline & $\mathrm{D}$ & 0.010 \\
\hline
\end{tabular}

Table 2. MUAV hardware specification

\begin{tabular}{|l|l|}
\hline \multicolumn{1}{|c|}{ Component } & \multicolumn{1}{c|}{ Ratings } \\
\hline Motors \& ESC & $\begin{array}{l}850 \mathrm{KV} \text { Brushless Out Runner / 20 } \\
\text { A }\end{array}$ \\
\hline Processor & AVR ATMega2560 $-16 \mathrm{MHz}$ \\
\hline Control Interface & $2.4 \mathrm{GHz}-9 \mathrm{Channel}$ \\
\hline Telemetry Date Interface & $433 \mathrm{MHz}$ RF \\
\hline \multicolumn{1}{|c|}{ Image Data Interface } & $5.8 \mathrm{GHz}$ Radio \\
\hline GPS & Ublox 10Hz \\
\hline Frame Type & $550 \mathrm{~mm}$ Glass Fiber \\
\hline
\end{tabular}

\section{RESULTS AND DISCUSSION}

MATLAB 2012a software is extensively used for the study of Firefly algorithm and graylevel images. The algorithm has been executed at a base station in a Windows 7 platform having 4GB RAM, Intel Core i7 $2.80 \mathrm{GHz}$ processor. Numerous gray-level images are used to test the proposed method. Results obtained using Firefly algorithm based image enhancement technique is shown in Figure 3. Where, Figures 3a-d are the experimental images which are enhanced using FA as illustrated in Figures 3e-h. For a detailed understanding of the proposed algorithm; Figure 3.a has been chosen to represent its corresponding histograms as shown in Figure 4. While Figure 5 shows the position of Fireflies in different Iterations during optimization of $\lambda$ and $\mu$ for Figure $3 a$. 


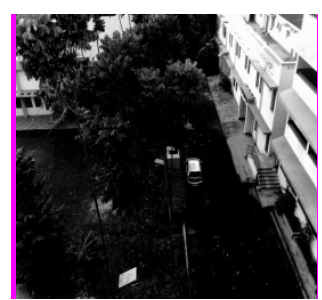

a)

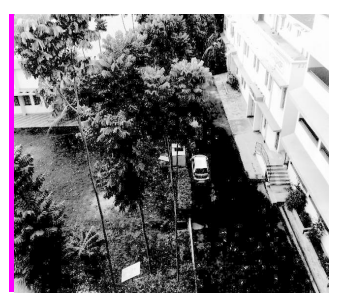

e)

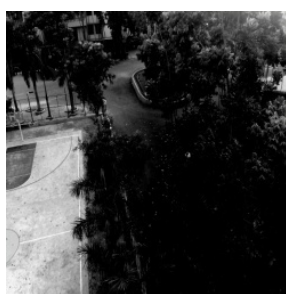

b)

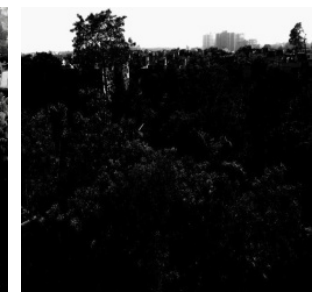

c)

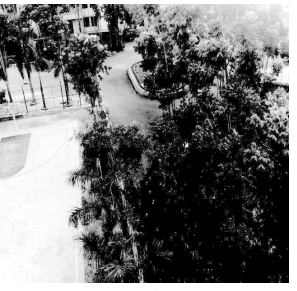

f)

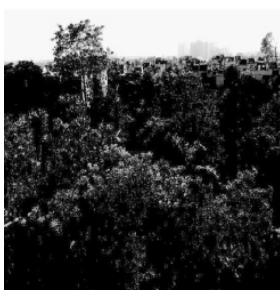

g)

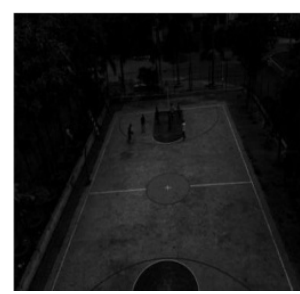

d)

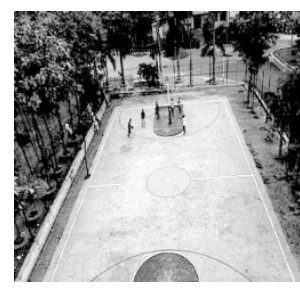

h)

Figure 3. Reports couple of FA based image enhancement results
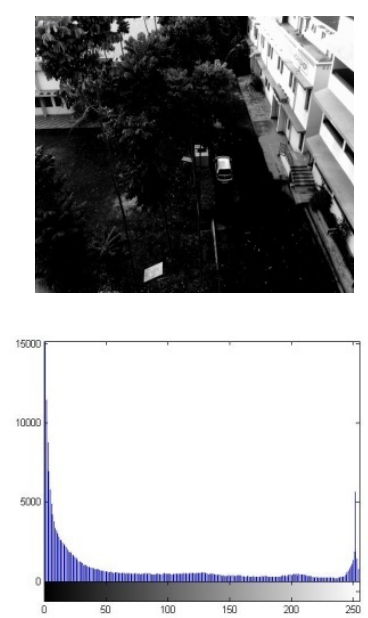

(a)
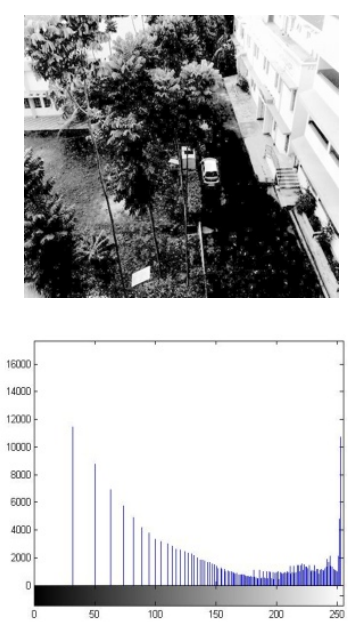

(b)

Figure 4. (a) Original image using FA and its corresponding histogram, (b) The enhanced image using FA and corresponding histograms 


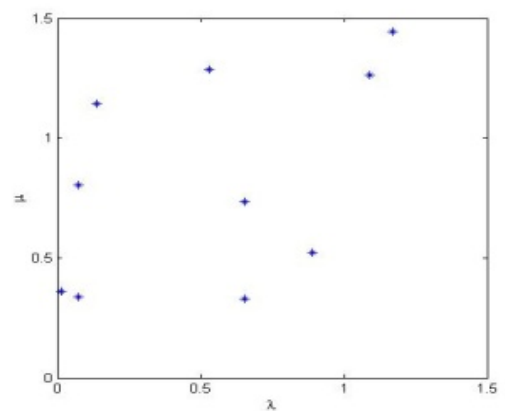

a) $1^{\text {st }}$ Iteration

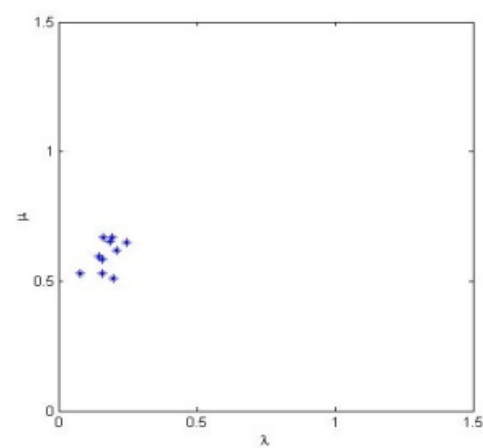

c) $10^{\text {th }}$ Iteration

Figure 5. Position of Fireflies in different Iteration

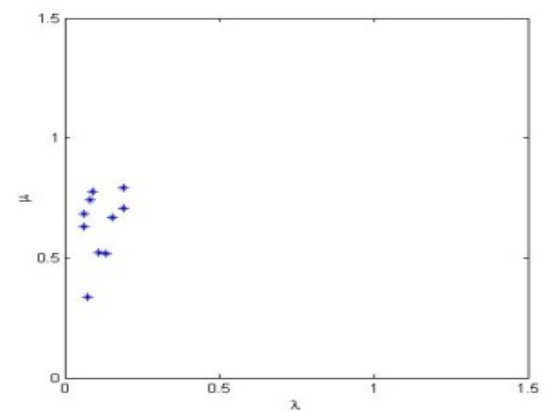

b) $5^{\text {th }}$ Iteration

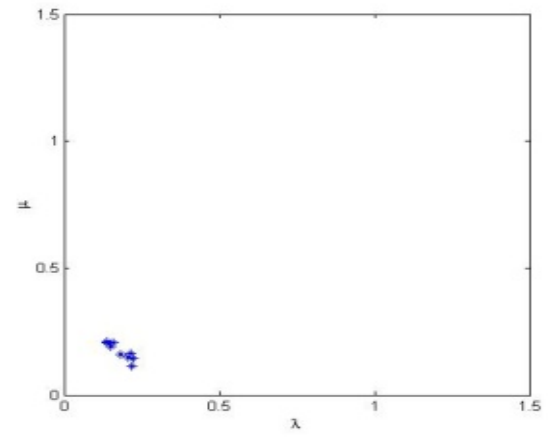

d) $25^{\text {th }}$ Iteration

\subsection{Comparing the results of Firefly algorithm versus PSO Algorithm}

A Comparison is done to benchmark Firefly algorithm used in this paper with the particle swarm optimization based approach. Table 3 shows a comparison between the fitness using PSO and Firefly algorithm to optimize the two parameters $\mu$ and $\lambda$. This table illustrates that the fitness of the firefly is better than that obtained using PSO for each corresponding iteration where,

1- Using FA, after the 16th iteration the fitness value is converged, and upon further iterations, it has produced the same fitness value.

2- Using PSO, it does not converge up to the 25th iteration also the fitness value of the 25th iteration is less than that obtained using FA.

Therefore, using FA is superior to PSO in terms of both better fitness value and less time consuming to converge.

Up to 25 iterations are shown in Table 3 and the obtained convergence graph is shown in Figure 6. The graph reports the No. of Iteration versus PSO and FA for the two parameter values as well as the fitness. 
Table 3. Comparison between PSO and Firefly

\begin{tabular}{|c|c|c|c|c|c|c|}
\hline \multirow[b]{2}{*}{ Iteration } & \multicolumn{3}{|c|}{ Particle Swarm Optimization } & \multicolumn{3}{|c|}{ Firefly Algorithm } \\
\hline & $\begin{array}{c}\text { Value of } \\
\lambda\end{array}$ & $\begin{array}{c}\text { Value of } \\
\qquad \mu\end{array}$ & Fitness & $\begin{array}{c}\text { Value of } \\
\lambda\end{array}$ & $\begin{array}{c}\text { Value of } \\
\qquad \mu\end{array}$ & Fitness \\
\hline 1 & 0.106262 & 0.992738 & 0.683074 & 0.007213 & 0.022342 & 0.413813 \\
\hline 2 & 0.141833 & 0.633116 & 0.778596 & 0.17992 & 0.099355 & 0.886541 \\
\hline 3 & 0.168203 & 0.454936 & 0.82557 & 0.17992 & 0.099355 & 0.886541 \\
\hline 4 & 0.143078 & 0.31382 & 0.835577 & 0.17992 & 0.099355 & 0.886541 \\
\hline 5 & 0.174575 & 0.224701 & 0.883726 & 0.15643 & 0.136084 & 0.889119 \\
\hline 6 & 0.134927 & 0.152609 & 0.844378 & 0.15643 & 0.136084 & 0.889119 \\
\hline 7 & 0.115409 & 0.109679 & 0.836878 & 0.170344 & 0.136651 & 0.890825 \\
\hline 8 & 0.119054 & 0.129758 & 0.826594 & 0.170344 & 0.136651 & 0.890825 \\
\hline 9 & 0.125812 & 0.149712 & 0.836487 & 0.170344 & 0.136651 & 0.890825 \\
\hline 10 & 0.12325 & 0.156228 & 0.833496 & 0.182495 & 0.153807 & 0.897810 \\
\hline 11 & 0.128806 & 0.171209 & 0.842316 & 0.167736 & 0.150865 & 0.901324 \\
\hline 12 & 0.149316 & 0.194893 & 0.863039 & 0.167736 & 0.150865 & 0.901324 \\
\hline 13 & 0.140327 & 0.18413 & 0.85256 & 0.167736 & 0.150865 & 0.901324 \\
\hline 14 & 0.145006 & 0.188058 & 0.856908 & 0.167736 & 0.150865 & 0.901324 \\
\hline 15 & 0.151556 & 0.195527 & 0.86337 & 0.167736 & 0.150865 & 0.901324 \\
\hline 16 & 0.140851 & 0.181527 & 0.853717 & 0.167736 & 0.150865 & 0.901324 \\
\hline 17 & 0.142395 & 0.183297 & 0.856259 & 0.180398 & 0.158748 & 0.908621 \\
\hline 18 & 0.120369 & 0.154993 & 0.830687 & 0.180398 & 0.158748 & 0.908621 \\
\hline 19 & 0.126402 & 0.162732 & 0.836943 & 0.180398 & 0.158748 & 0.908621 \\
\hline 20 & 0.130192 & 0.167606 & 0.842138 & 0.180398 & 0.158748 & 0.908621 \\
\hline 21 & 0.141229 & 0.181795 & 0.853513 & 0.180398 & 0.158748 & 0.908621 \\
\hline 22 & 0.138157 & 0.177827 & 0.851071 & 0.180398 & 0.158748 & 0.908621 \\
\hline 23 & 0.131376 & 0.169096 & 0.842482 & 0.180398 & 0.158748 & 0.908621 \\
\hline 24 & 0.121401 & 0.156256 & 0.83059 & 0.180398 & 0.158748 & 0.908621 \\
\hline 25 & 0.121887 & 0.156884 & 0.831701 & 0.180398 & 0.158748 & 0.908621 \\
\hline
\end{tabular}




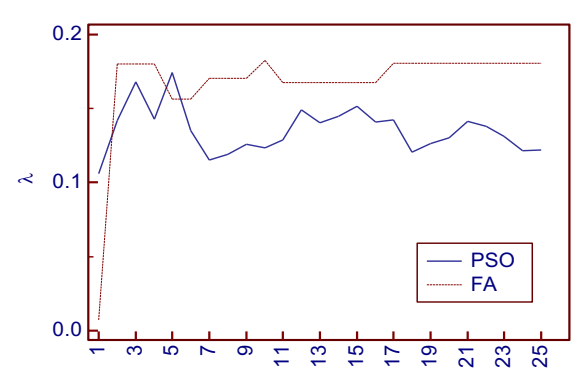

No. of Iteration

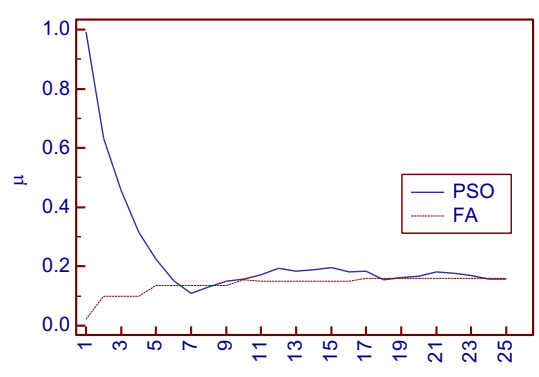

No. of iteration

a) Value of $\lambda$

b) Value of $\mu$

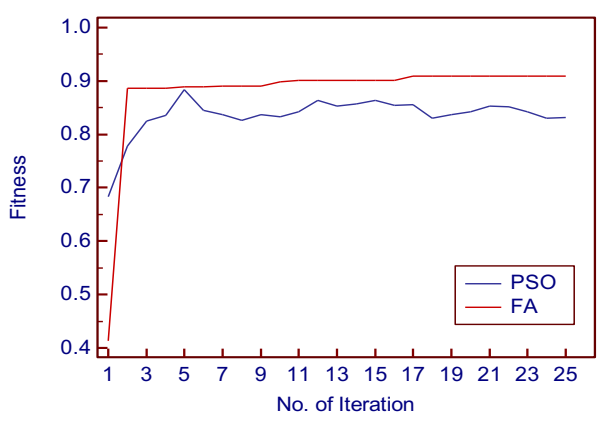

c) Value of Fitness

Figure 6. Convergence graph using PSO and FA

\subsection{Image Quality Analysis}

Image quality analysis or assessment can be used for modeling the metric between an original image and an enhanced version of it. The goal is to evaluate and compare the performance of image processing algorithms. Therefore, image quality analysis is a procedure to impose a grading based on various parameters that affects the image quality. It reflects the differences between corresponding pixels in the original and the degraded image. According to National Imagery Interpretability Rating Scale (NIIRS) [30], image quality can be viewed as a task-based scale used for military, civilian, and agricultural applications. The scale initiates from 0 (implies poorest quality) and ends with 9 (implies highest quality). The image acquired by the aerial digital camera is supposed to be more powerful information for remote sensing. Due to enhancement of image the quality improved. Table 4 demonstrates the IQA metrics that measure the image quality and their equations. Consequently, Table 5 compares the image quality of both FA and PSO algorithms using these IQA metrics. 
Table 4. Image Quality Analysis Measures (IQA)

\begin{tabular}{cc}
\hline IQA Measures & Equation \\
Mean Square Error & $M E=\frac{1}{M N} \sum_{j=1}^{M} \sum_{i=1}^{N}\left(x_{j, k}-x_{j, k}^{\prime}\right)^{2}$ \\
Average Difference & $A D=\sum_{j=1}^{M} \sum_{k=1}^{N}\left(x_{j, k}-x_{j, k}^{\prime}\right) \quad M N$ \\
Structural Content & $S C=\sum_{j=1}^{M} \sum_{k=1}^{N} x_{j, k}^{2} \quad S C=\sum_{j=1}^{M} \sum_{k=1}^{N} x_{j, k}^{\prime 2}$ \\
Maximum Difference & $M D=\operatorname{Max}\left(\left|x_{j, k}-x_{j, k}^{\prime}\right|\right)$ \\
Peak Signal to Noise ratio & PSNR $=10 \log _{10}\left(255^{2 / \mathrm{MSE})}\right.$
\end{tabular}

Table 5. Different Values of IQA parameters

\begin{tabular}{ccccccc}
\hline $\begin{array}{c}\text { Image } \\
\text { No }\end{array}$ & & $\begin{array}{c}\text { Mean } \\
\text { Square } \\
\text { Error }\end{array}$ & $\begin{array}{c}\text { Average } \\
\text { Difference }\end{array}$ & $\begin{array}{c}\text { Structural } \\
\text { Content }\end{array}$ & $\begin{array}{c}\text { Maximum } \\
\text { Difference }\end{array}$ & $\begin{array}{c}\text { Peak } \\
\text { Signal to } \\
\text { Noise ratio }\end{array}$ \\
\hline Fig 3.e & FA & 5397.549 & -53.2649 & 0.428894 & 21 & 10.80884 \\
& PSO & 2052.664 & -19.4487 & 0.941216 & 94 & 15.00763 \\
Fig 3.f & FA & 5397.549 & -53.2649 & 0.428894 & 21 & 10.80884 \\
& PSO & 2052.664 & -19.4487 & 0.941216 & 94 & 15.00763 \\
Fig 3.g & FA & 2663.231 & -30.7066 & 0.669474 & 26 & 13.87671 \\
& PSO & 2352.484 & -27.1597 & 0.743087 & 35 & 14.41554 \\
\multirow{2}{*}{ Fig 3.h } & FA & 17135.15 & -113.412 & 0.03226 & 1 & 5.791924 \\
& PSO & 21283.47 & -126.248 & 0.02689 & 1 & 4.85038 \\
\hline
\end{tabular}

Note that for both log transform based image enhancement techniques (FA and PSO), PSNR, MSE or any IQA factors are not taken into consideration for the computation of the fitness function. IQA, an independent study reported at the end of our paper, assesses the contrast improvement of the experimental images.

Table 5 clearly depicts that PSO based technique sometimes gives higher PSNR and less MSE than FA for the current experimental setup (because of selection of images, parameter setting, no. of fireflies, etc.). Whereas, Table 3 clearly shows that for the image in Figure 3a, FA based approach gives higher fitness than PSO based technique in all iterations and $\lambda, \mu$ and fitness value converges for FA based after $16^{\text {th }}$ iteration. On the other hand, PSO does not converge even after 25 iterations (Table 3). All experimental images are tested to report the robustness of FA based approach in terms of speed giving similar results.

Therefore, the motivation of selecting the FA based approach is that it has faster convergence rate and better fitness (Table 3) than that of PSO based approach, often slightly compromising with the image quality. The proposed approach established its 
efficiency, thus, it is recommended to include different optimization algorithms. In addition, a comparative study of including canny detector with the results obtained with sobel detector can be carried out in the future work.

\section{CONCLUSION}

The Mini Unmanned Aerial Vehicle (MUAV) system used in this work is a highly robust test bed. Although the application of UAV is very common in military and civilian applications nowadays, but it's having some disadvantages. The UAV like multi-rotor systems, mostly suffer from low endurance time, so for a long range mission some alternatives have to be chosen. Due to heavy rain, high storm the performance of the UAV might be degraded due to aerial instability and poor satellite geometry. In spite of all these disadvantages a MUAV platform; is used for aerial image acquisition because of its position hold quality.

In this paper, a MUAV system is proposed that capture an aerial photograph for situation where it gets a low contrast/quality image. Then a Firefly algorithm based automatic image enhancement method for gray level images is applied. Using FA is superior to PSO with respect to the convergence rate and the fitness values. As convergence is the outcome of meta-heuristic framework. Therefore, this work can be further extended using Multiobjective optimization techniques and can be compared with the obtained Firefly algorithm based results.

Apart from Firefly Algorithm there are several other meta-heuristic algorithms, namely: Cuckoo search (CS), Bat algorithm, etc., which can be applied for the enhancement purpose which is beyond the scope of this study.

\section{REFERENCES}

1. R. Yanushevsky, Guidance of Unmanned Aerial Vehicles: CRC Press, (2011).

2. H. Duan, X. Zhang and C. Xu, Bio-Inspired Computing, Beijing, China: Science Press, (2011).

3. G. Wang, L. Guo, H. Duan, L. Liu, H. Wang and M. Shao, Path planning for uninhabited combat aerial vehicle using hybrid meta-heuristic DE/BBO algorithm. Advanced Science, Engineering and Medicine 4(6) (2012) 550-564.

4. Y. Pehlivanoglu, A new vibrational genetic algorithm enhanced with a Voronoi diagram for path planning of autonomous UAV, Aerospace Science and Technology 16 (2012) 47-55.

5. W. Ye, D. Ma and H. Fan, Algorithm for low altitude penetration aircraft path planning with improved ant colony algorithm, Chinese Journal of Aeronautics 18(4) (2005) 304-309.

6. H. Duan, Y. Yu, X. Zhang and S. Shao, Three-dimension path planning for UCAV using hybrid meta-heuristic ACO-DE algorithm, Simulation Modeling Practice and Theory 18(8) (2010)1104-1115.

7. G. Wang, L. Guo, H. Duan, L. Liu, H. Wang and B. Wang, A hybrid meta-heuristic DE/CS algorithm for UCAV path planning, Journal of Information and Computational Science 5(2012) 4811-4818. 
8. O. Haddad, A. Afshar and M. Marino, Honey-Bees Mating Optimization (HBMO) Algorithm: A New Heuristic Approach for Water Resources Optimization, Journal of Water Resources Management 20(5)(2006) 661-680.

9. G. Kumar and B. Harish, Particle Swarm Optimization (PSO) Technique for Image Enhancement, International Journal of electronics \& communication technology (IJECT) 4(3) (2013).

10. A. Gandomi, X.Yang, S. Talatahari and A. Alavi, Firefly algorithm with chaos. Communications in Nonlinear Science and Numerical Simulation 18(1) (2013) 89-98.

11. S. Pal and A. Rosenfeld, Image enhancement and thresholding by optimization of fuzzy compactness, Pattern Recognition Letters 7(1988) 77-86.

12. C. Munteanu and A. Rosa, Automatic image enhancement technique using genetic algorithms, Proceedings of the 2000Congress, Evolutionary Computation, La Jolla, CA 2(2000) 1535 - 1542.

13. S. Mitra, C. Murthy and M. Kundu, Image magnification technique using partitioned iterative function system, Pattern Recognition 33(2000) 1119-1133.

14. D. Gibbins, P. Roberts and L. Swierkowski, A video geo-location and image enhancement tool for small unmanned air vehicles (UAVs), Processing Conference Intelligent Sensors, Sensor Networks and Information (2004) 469-473.

15. M. Braik, A. Sheta and A. Ayesh, Image Enhancement technique using Particle Swarm Optimization, Proceedings of the World Congress on Engineering 2007(WCE 2007), July 2 - 4, London, U.K I (2007).

16. L.Yuping and G. Medioni, Map-Enhanced UAV Image Sequence Registration and Synchronization of Multiple Image Sequences, IEEE Conference on Computer Vision and pattern recognition (CVPR '07), Minneapolis, MN (2007) 1-7.

17. G. Apurba and G. Ashish, Grayscale image enhancement technique by Particle Swarm Optimization, 2009 World Congress on Nature \& Biologically Inspired Computing (NaBIC 2009)(2009).

18. R. Tirimula, Novel approach to Image Edge Enhancement using Ant Colony Optimization algorithm for Hybridized Smoothening Filters, International Conference on Advances \& Emerging Trends in Computing Technologies (ICAET-2010), SRM University, Chennai, India (2010).

19. K.Venkateshwarlu and B. Anju, Image enhancement technique using Fuzzy system inference, Recent trends in Mathematical Sciences, Osmania University, Hyderabad, India (2010).

20. C. Hanxing and T. Jun, A novel image enhancement method using Particle Swarm Optimization, International Conference in Uncertainty Reasoning and Knowledge Engineering (URKE) (2011).

21. T. Hassanzadeh, H. Vojodi and F. Mahmoudi, Non-linear Grayscale Image Enhancement Based on Firefly Algorithm, Swarm, Evolutionary and Memetic Computing (2011) 174-181.

22. G. Kanika and G. Akshu, Image Enhancement technique using Ant Colony Optimization, IOSR Journal of VLSI and Signal Processing (IOSR-JVSP)1(3) (2012) 38-45.

23. Su. Xiaoping, F. Wei, Sh. Qing, H. Xiulan, Image enhancement method using the QuantumBehaved Particle Swarm Optimization with an Adaptive Strategy, Mathematical Problems in Engineering 2013(2013). 
24. G. Kumar and B. Harish, Particle Swarm Optimization (PSO) Technique for Image Enhancement, Proceedings of Seventh International Conference on Bio-Inspired Computing: Theories and Applications (IJECT) 4(2013).

25. S. Nadezhda and S. Stephanie, Fast and efficient iris image enhancement using logarithmic image processing, Proceedings of the SPIE 7667(2010).

26. S. Lange, S. Niko and P. Peter, Autonomous landing for a multirotor UAV using vision, International Conference on Simulation, Modeling, and Programming for Autonomous Robots (SIMPAR 2008) (2008) 482-491.

27. X. Yang, Firefly algorithm, stochastic test functions and design optimization, International Journal of Bio-Inspired Computation 2(2) (2010) 78-84.

28. N. Dey, S. Sourav, C. Sayan, D. Achintya, S. Sheli, and S. Jasjit, Firefly Algorithm for Optimization of Scaling Factors During Embedding of Manifold Medical Information: An Application in Ophthalmology Imaging, Journal of Medical Imaging and health informatics 4(3) (2014) 384-394.

29. X. Yang and X. He, Firefly Algorithm: Recent Advances and Applications, Int. J. Swarm Intelligence 1(1) (2013) 36-50.

30. J. Irvine, National imagery interpretability rating scales (NIIRS): overview and methodology. International Society for Optics and Photonics, Optical Science, Engineering and Instrumentation'97(2013) 93-103. 